\title{
Reliable Estimates of Planetary and Solar Magnetic Fields - A Case for Abundance of Hydrogen in the Earth's Core
}

\author{
By \\ Fritz Paschke \\ (Vorgelegt in der Sitzung der math.-nat. Klasse am 15. November 2007 \\ durch das w. M. Fritz Paschke)
}

\begin{abstract}
A theory of planetary magnetic fields published in 1998, which avoids the drawbacks of the conventional dynamo theories and is based on rotating dipole domains, is supplemented by adding data for the Sun and young Mars. Acceptable accuracy is achieved in the prediction of magnetic moments spanning 11 orders of magnitude for celestial bodies differing in mass by a factor of $6 \cdot 10^{6}$. One of the weaknesses of the theory lies in the fact that in assuming iron to be the dominant element in the Earth's core, inacceptable temperature levels are necessary to support the dipole model. However, on the basis of later reports it may be concluded that metallic hydrogen is present in the Earth's core in a higher concentration than assumed earlier. Taking the presence of hydrogen into account, the temperatures needed to support the dipole model are reduced to widely accepted levels, provided that the ratio of hydrogen on the total mass density is at least $1 / 6$ (calculated for a valency of the iron nuclei of $Z=1$ ) or at least $1 / 10$ (estimated for $Z=2$ ). Thus Earth-like planets appear closer in structure to the gas planets than assumed hitherto. A loss of hydrogen in the core leads to the extinction of the magnetic field - a fate, which may have been suffered by Mars.
\end{abstract}

\section{Introduction}

The model used in ref. [1] is based on considering a slab of conducting liquid keeping together by a binding energy represented in Fig. 1 by a potential wall. 

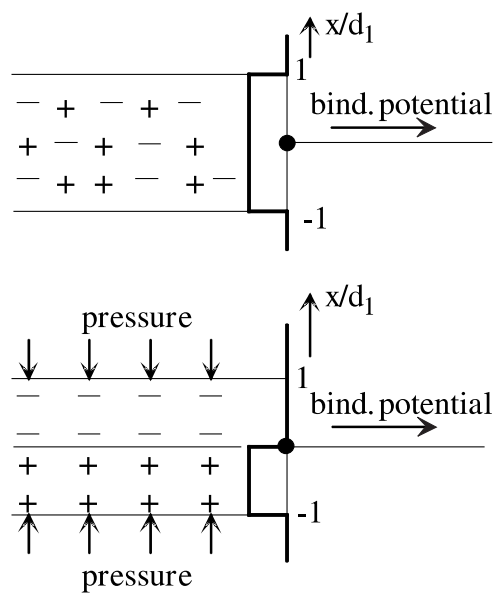

Fig. 1. Upper part: Conducting plasma layer of thickness $2 d_{1}$, binding energy symbolized by potential barriers. Lower part: Plasma layer under high pressure, forming dipole domains. Here $d_{1}$ is 3,3 times the Debye-distance (Eq. (19) in ref. [1]), calculated for the average particle density

The assumption made to obtain a loss-free model is that under high pressure the electrons separate from the densely packed atomic nuclei and form a dipole layer. The large force due to electric space-charge fields is balanced by pressure gradients as indicated for a valency of $Z=1$ in Figs. 2 and 3.

To prevent back-diffusion of the electrons, the potential barrier has to be maintained at a level $\gg k T / e$, with $k$ as Boltzmann's constant,

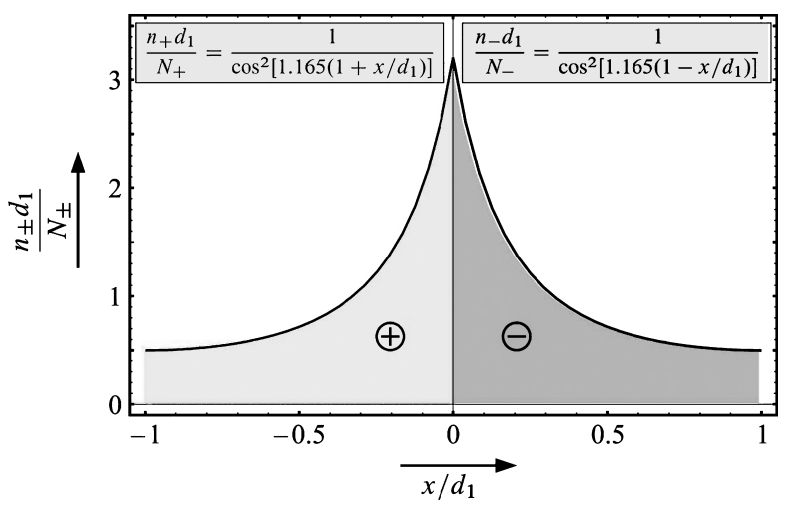

Fig. 2. Distribution of particle densities within the dipole domains relative to the average particle density (Eqs. (18) and (10) of ref. [1]), with $N_{ \pm}$as particle density per area 


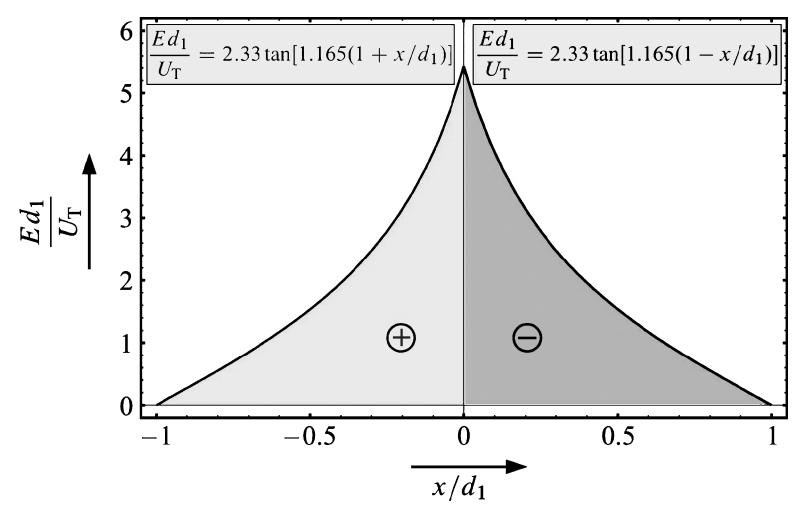

Fig. 3. Distribution of the electric field within the dipole domains (Eqs. (7) and (9) of ref. [1]), normalized to $U_{T} / d_{1}$

$T$ as absolute temperature and $e$ as elementary electric charge. Note that the dipole layer maintains neutrality towards the outside (zero external electric field).

When stacking up such dipole layers onion-like for the planetary cores and attributing to the particles an additional degree of freedom based on the stored electrical energy per particle, the following formulas for the magnetic moments $m_{H}$ had been derived in ref. [1].

For a spherical shell of thickness $d a$ at the radius $a$ (Eq. (28) in ref. [1])

$$
\frac{d m_{H}}{2 \pi}=-1.5 \Omega \cdot \sqrt{p_{0} \varepsilon} \cdot a^{3} d a .
$$

Here $\varepsilon$ is the permittivity, $\Omega$ is the angular velocity (for Earth $\Omega>0$ ) and $p_{0}$ is the pressure, which is approximated by the parabolic function (Eq. (29) in ref. [1])

$$
p_{0}(a)=p_{0}(0)-\left(\frac{a}{a_{1}}\right)^{2}\left(p_{0}(0)-p_{0}\left(a_{1}\right)\right)
$$

with $a_{1}$ the outer radius of the core. Integrating Eq. (1) with Eq. (2) over the core yields the total magnetic dipole moment (Eq. (31) in ref. [1])

$$
\begin{aligned}
\frac{m_{H}}{2 \pi}= & -\frac{8 \Omega}{3}\left(\frac{\varepsilon p_{0}(0)}{2}\right)^{1 / 2} \cdot 0.8 a_{1}^{4} \\
& \times \frac{\frac{1}{3}\left[1-\left(\frac{p_{0}\left(a_{1}\right)}{p_{0}(0)}\right)^{3 / 2}\right]-\frac{1}{5}\left[1-\left(\frac{p_{0}\left(a_{1}\right)}{p_{0}(0)}\right)^{5 / 2}\right]}{\left(1-\frac{p_{0}\left(a_{1}\right)}{p_{0}(0)}\right)^{2}} .
\end{aligned}
$$


For the signs in Eqs. (1) and (3) to be valid, it is assumed that the outermost layer is composed of electrons. If the dipole orientation is reversed (ions in the outermost layer) the field is reversed.

Eqs. (1) and (2) allow the calculation of the magnetic fields. With

$$
H_{0}=-\frac{8}{3} \cdot \Omega \cdot\left(\frac{\varepsilon p_{0}(0)}{2}\right)^{1 / 2} \cdot 0.8 a_{1}
$$

and the normalized radius

$$
\rho=\frac{r}{a_{1}}
$$

the distributions in spherical coordinates for $\rho \leq 1 \mathrm{read}$

$$
\begin{aligned}
H_{r}= & H_{0} \cdot \cos \vartheta\left\{\frac{1}{k^{2} \rho^{3}}\left[\frac{1}{3}\left[1-\left(1-\rho^{2} k\right)^{3 / 2}\right]-\frac{1}{5}\left[1-\left(1-\rho^{2} k\right)^{5 / 2}\right]\right]\right. \\
& \left.+\frac{1}{2 k}\left[\arcsin k-\arcsin \rho k+k(1-k)^{1 / 2}-\rho k\left(1-\rho^{2} k\right)^{1 / 2}\right]\right\}, \\
H_{\vartheta}= & \frac{1}{2} \cdot H_{0} \cdot \sin \vartheta \cdot\left\{\frac{1}{k^{2} \rho^{3}}\left[\frac{1}{3}\left[1-\left(1-\rho^{2} k\right)^{3 / 2}\right]-\frac{1}{5}\left[1-\left(1-\rho^{2} k\right)^{5 / 2}\right]\right]\right. \\
& \left.-\frac{1}{k}\left[\arcsin k-\arcsin \rho k+k(1-k)^{1 / 2}-\rho k\left(1-\rho^{2} k\right)^{1 / 2}\right]\right\}
\end{aligned}
$$

and for $\rho \geq 1$

$$
\begin{aligned}
& H_{r}=H_{0} \cdot \cos \vartheta \cdot \frac{1}{\rho^{3} k^{2}}\left[\frac{1}{3}\left[1-(1-k)^{3 / 2}\right]-\frac{1}{5}\left[1-(1-k)^{5 / 2}\right]\right], \\
& H_{\vartheta}=H_{0} \cdot \sin \vartheta \cdot \frac{1}{2 \rho^{3} k^{2}}\left[\frac{1}{3}\left[1-(1-k)^{3 / 2}\right]-\frac{1}{5}\left[1-(1-k)^{5 / 2}\right]\right] .
\end{aligned}
$$

Here, not to be confounded with Boltzmann's constant introduced later,

$$
k=1-\frac{p_{0}\left(a_{1}\right)}{p_{0}(0)} .
$$

For Earth, $k=0.6$ and the field distribution of Fig. 4 is obtained, with the magnetic south pole at the geographic north pole. The energy of the Earth magnetic field is calculated to be $4.563 \cdot 10^{18}$ Joule, $14.3 \%$ of which is stored outside of the core. 


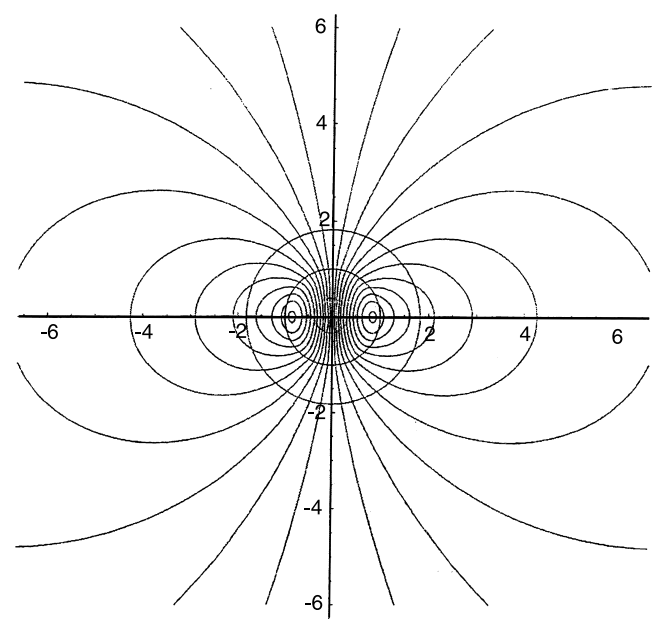

Fig. 4. Field lines, calculated from Eqs. (6) and (7) for Earth, with the radii relative to the outer core radius

\section{Magnetic Moments in the Solar System}

Eq. (7a) leads to an Earth magnetic field at the poles of about $24 \mathrm{~A} / \mathrm{m}$, which corresponds to an induction of $30 \mu \mathrm{T}$ (0.3 Gauss); this is close to the observed values. When applying the model to other planets, the lack of knowledge of their internal structures is a problem which had been tried to overcome by using a two-shell model with a core material of average mass-density $\rho_{1}$ and a mantle material of average mass-density $\rho_{2}$. With plausible values for $\rho_{1,2}$ and the known values of radii and masses, the parameters entering Eq. (3) can be determined. Confidence in this method is gained by applying it to Earth, where the two-shell model leads to an error of only $2 \%$ in magnetic moment as compared to the result gained from the more accurate data available from seismic measurements.

The values of the mass densities used were:

$$
\begin{array}{lll}
\text { for Earth and Venus } & \rho_{1}=1.2 \cdot 10^{4} \mathrm{~kg} / \mathrm{m}^{3}, & \rho_{2}=4.22 \cdot 10^{3} \mathrm{~kg} / \mathrm{m}^{3} ; \\
\text { for Mercury and Mars } & \rho_{1}=10^{4} \mathrm{~kg} / \mathrm{m}^{3}, & \rho_{2}=3 \cdot 10^{3} \mathrm{~kg} / \mathrm{m}^{3} ; \\
\text { for Jupiter and Saturn } & \rho_{1}=1.4 \cdot 10^{4} \mathrm{~kg} / \mathrm{m}^{3}, & \rho_{2}=0.55 \cdot 10^{3} \mathrm{~kg} / \mathrm{m}^{3} ; \\
\text { for Uranus and Neptune } & \rho_{1}=1.3 \cdot 10^{4} \mathrm{~kg} / \mathrm{m}^{3}, & \rho_{2}=0.8 \cdot 10^{3} \mathrm{~kg} / \mathrm{m}^{3}, \\
\text { for Sun } & \rho_{1}=1.3 \cdot 10^{5} \mathrm{~kg} / \mathrm{m}^{3}, & \rho_{2} \ll \rho_{1} .
\end{array}
$$


Table 1. $a_{1} / 10^{6} \mathrm{~m}$ is the core radius in $10^{6}$ Meters, $p\left(a_{1}\right) / 10^{11} \mathrm{P}$ and $p(0) / 10^{11} \mathrm{P}$ are the pressures at the edge and the center of the core in $10^{11}$ Pascal, $\Omega / \Omega_{E}$ the angular velocity of the planet related to the Earth's value, and $m_{H} / m_{H E}$ the magnetic dipole moment related to the Earth's value

\begin{tabular}{|c|c|c|c|c|c|c|}
\hline & $\frac{a_{1}}{10^{6} \mathrm{~m}}$ & $\frac{p\left(a_{1}\right)}{10^{11} \mathrm{P}}$ & $\frac{p_{0}}{10^{11} \mathrm{P}}$ & $\frac{\Omega}{\Omega_{E}}$ & $\frac{m_{H}}{m_{H E}}$ theor. & $\begin{array}{l}\frac{m_{H}}{m_{H E}} \text { observed, } \\
\text { from NESS [2] }\end{array}$ \\
\hline Mercury & 1.71 & 0.0633 & 0.513 & $1.7 \cdot 10^{2}$ & $2 \cdot 10^{-4}$ & $6.25 \cdot 10^{-4}$ \\
\hline Venus & 3.0 & 1.09 & 3.14 & $4.1 \cdot 10^{-3}$ & $2.5 \cdot 10^{-3} ? / 0$ & $<5 \cdot 10^{-5}$ \\
\hline Earth & 3.5 & 1.21 & 4.6 & 1 & 1 & 1 \\
\hline Mars & 1.7 & 0.2 & 0.6 & 0.975 & $2 \cdot 10^{-2}$ & $\begin{array}{r}\left(<2.5 \cdot 10^{-4}\right. \\
\text { today }) \\
2 \cdot 10^{-2} \text { from } \\
\text { Mars Global } \\
\text { Surveyor for } \\
\text { young Mars }\end{array}$ \\
\hline Jupiter & 26.5 & 8.61 & 200 & 2.41 & $4.2 \cdot 10^{4}$ & $2 \cdot 10^{4}$ \\
\hline Saturn & 10.7 & 3.41 & 34.6 & 2.24 & 463 & 590 \\
\hline Uranus & 8.28 & 1.8 & 17.9 & 1.39 & 74.3 & 47.5 \\
\hline Neptune & 9.82 & 2.07 & 24.7 & 1.48 & 180 & 25 \\
\hline Sun & 152.4 & $\ll p(0)$ & $5.48 \cdot 10^{5}$ & $4 \cdot 10^{-2}$ & $2.18 \cdot 10^{7}$ & $\begin{array}{l}10^{7} \text { (from } \\
\quad \text { ref. [3]) }\end{array}$ \\
\hline
\end{tabular}

Table 1 summarizes the results for the solar system (excluding Pluto). No attempt has been made to improve agreement by adopting other values for $\rho_{1,2}$ than those given above. The agreement of observed (N. F. NESS [2]) and calculated values spanning 11 orders of magnitude for celestial bodies differing in mass by $6 \cdot 10^{6}$ is remarkable. The data given for the Sun refer to the dipole-part of its magnetic field (FRIEDMANN [3], MERRILL et al. [4]). Also remarkable appears the fact that, based on observations of the Global Surveyor (CONNERNEY et al. [5]), young Mars had exactly the magnetic moment predicted by the theory, but lost it later for unknown reasons. For Venus, the rotational speed $(<0.9 \mathrm{~m} / \mathrm{s})$ is well below the thermal velocities, so that the magnetic moment can be assumed to be zero - Eq. (3) is not applicable.

\section{The Temperature Dilemma}

The theory suffers from the drawback that very high core temperatures are necessary to support the dipole model. For a valency of $Z=1$ and predominating iron, the temperature (from Eq. (34) in ref. [1]) reads

$$
T=\frac{m \cdot p_{0}}{\rho_{m} \cdot k} \cdot \frac{2}{1+Z}
$$


with $\rho_{m}$ as mass density, $m$ as atomic mass (iron for Earth), $p_{0}$ as pressure, $Z$ as valency and $k$ as Boltzmann's constant. With $m=$ $9.37 \cdot 10^{-26} \mathrm{~kg}, \rho_{m} \doteq 10^{4} \mathrm{~kg} / \mathrm{m}^{3}, p_{0}=1.6 \cdot 10^{11} \mathrm{P}-$ the value at the outer edge of the Earth's core - and a valency of $Z=1$, an unreasonable value of $T=109,020$ Kelvin is obtained. A great dilemma for the theorist, who may be rescued by well-based arguments (WILLIAMS and HEMLEY [6]) for an abundance of hydrogen in the core.

Thus Eq. (9) has been re-examined for a mixture of iron and hydrogen. The field- and charge distributions, calculated in ref. [1], are valid for a mixture only for equal valency of the components. Thus a valency of $Z=1$ had to be assumed for the iron nuclei. Taking $0 \leq \gamma \leq 1$ as fraction of the hydrogen part on the total mass density,

$$
\rho_{m}=\frac{m N_{+}}{d_{1}} \cdot \frac{1}{1+\gamma\left(\frac{m}{m_{p}}-1\right)}
$$

with $m_{p}$ as the proton mass and $N_{+} / d_{1}$ as average density of the nuclei, which, from Eqs. (12) and (15) of ref. [1], is given by

$$
\frac{N_{+}}{d_{1}}=\frac{2 p_{0}}{k T} .
$$

From Eqs. (10) and (11) the temperature becomes

$$
T=\frac{m p_{0}}{\rho_{m} \cdot k} \cdot \frac{1}{1+\gamma\left(\frac{m}{m_{p}}-1\right)} .
$$

The reduction factor for the temperature reaches the necessary value of (at least) 0.1 for $\gamma=0.16$ or $1 / 6$. If a reduction down to 5000 Kelvin were required, $\gamma \doteq 0.38$. For valencies of the iron nuclei exceeding 1, the necessary hydrogen concentration becomes lower, but cannot be analyzed rigorously on the basis of ref. [1]. But a crude estimate is given by Eq. (9), which yields a reduction factor for the temperature of $2 /(1+Z)$, so that with $Z=2, \gamma=0.1$ or $1 / 10$ is estimated for a reduction factor of 0.1 .

The high concentration of hydrogen postulated here appears possible in a plasma-state of the material. Metallic hydrogen caught the interest of scientists since a long time to explain, for example, the behaviour of gas planets or to seek practical applications (W. J. NeSS [7]). For the pressure inside of the Earth's core, a plasma state appears possible. Critical is the application for Mercury, where the pressure reaches only about $50 \mathrm{GP}$ (see Table 1), but metallic hydrogen is rich 
in unexpected properties (BABAEV et al. [8]), so that a mixture of iron and hydrogen may be suspected to be in a plasma state even at the pressures in Mercury's core.

The conclusions are:

- The theory of rotating dipole domains yields reliable estimates of the magnetic moments for Sun and all planets.

- For reasonable core temperatures, hydrogen has to appear in abundance in all Earth-like planets.

- For Mars, the loss of magnetic field may have been caused by a loss of hydrogen in its core.

\section{References}

[1] PASchKe, F. (1998) Rotating electric dipole domains as a loss-free model for the earth's magnetic field. Sitzungsber. Abt. II 207: 213-228; Transact. Austrian Academy of Sciences

[2] NESS, N. F. (1994) Intrinsic magnetic fields of the planets: Mercury to Neptune. Phil. Trans. R. Soc. London. Ser. A 349: 249-260

[3] Friedman, H. (1986) Sun and Earth. Sci. Americ. Books, New York

[4] Merrill, R. T., McElhinny, M. W., McFadden, P. L. (1998) The Magnetic Field of the Earth (Int. Geophys. Ser., Vol. 63). Academic Press, San Diego

[5] Connerney, J. E. P., Acuna, M. H., Wasilewski, P. J., Ness, N. F., RÈme, H., Mazelle, C., Vignes, D., Lin, R. P., Mitchell, D. L., Cloutier, P. A. (1999) Magnetic lineations in the ancient crust of mars. Science 284: 794-798

[6] Williams, Q., HEMley, R. J. (2001) Hydrogen in the deep earth. Ann. Rev. Earth Planet Sci. 29: $365-418$

[7] Ness, W. J. (1999) Metastable solid metallic hydrogen. Phil. Mag. B 79/4: 655-661

[8] BABAev, E., Sudbo, A., AshCroft, N. W. (2004) A superconductor to superfluid phase transition in liquid metallic hydrogen. Nature 431: 666-668

Author's address: Prof. Dr. Fritz Paschke, Kahlenberger Straße 35/2, 1190 Wien, Austria. E-Mail: fritz.paschke@chello.at. 